\title{
max \\ Blockchain-Based Auctioning for Energy Storage Sharing in a Smart Community
}

\author{
Uyikumhe Damisa (i) and Nnamdi I. Nwulu *(i)
}

Center for Cyber Physical Food, Energy and Water Systems (CCP-FEWS), University of Johannesburg,
Johannesburg 2092, South Africa; uyikumhe@yahoo.com
* Correspondence: nnwulu@uj.ac.za

\begin{abstract}
The increasing prevalence of renewable energy resources introduces a high variability that complicates the task of energy management in modern power grids. Among other technologies, batteries have proven effective in managing power imbalances in such grids. However, the high cost of large-scale batteries, coupled with their enormous space requirements, could deter their adoption by large consumers such as shared facility controllers. The aggregation of residential energy storage units offers shared facility controllers (SFCs) an alternative way to leverage storage; however, a secure scheme that promotes fairness and transparency in the selection and compensation of shared storage unit owners is needed. To this end, an Ethereum smart contract that makes residential storage capacities available to SFCs via a double auction mechanism is proposed. The contract is written with solidity and deployed in the browser-based Remix-integrated development environment. Scenario tests prove the effectiveness of the smart contract in selecting and compensating the owners of shared storage capacities, according to predefined auction rules.
\end{abstract}

Keywords: blockchain; smart contract; peer-to-peer trading; storage sharing

check for

updates

Citation: Damisa, U.; Nwulu, N.I. Blockchain-Based Auctioning for Energy Storage Sharing in a Smart Community. Energies 2022, 15, 1954. https://doi.org/10.3390/en15061954

Academic Editor: Abdul-Ghani Olabi

Received: 24 December 2021

Accepted: 14 February 2022

Published: 8 March 2022

Publisher's Note: MDPI stays neutral with regard to jurisdictional claims in published maps and institutional affiliations.

Copyright: () 2022 by the authors Licensee MDPI, Basel, Switzerland. This article is an open access article distributed under the terms and conditions of the Creative Commons Attribution (CC BY) license (https:/ / creativecommons.org/licenses/by/ $4.0 /)$.

\section{Introduction}

The influx of intermittent renewable energy generation into electric power grids gives rise to grid stability issues as grid planning and management become more complicated. This complication lies in the difficulty of accurately predicting the output of most types of renewable energy sources. Energy storage proves effective in handling the resulting energy imbalances. With its inherent ability to respond rapidly, storage can ensure grid stability during periods of power generation and consumption mismatch [1]. Energy storage is also crucial for the enhancement in the resilience and efficiency of smart grids and energy systems in general [2]. Residential storage units, in particular, can be harnessed for grid and consumer benefits. They can be optimally managed to reduce energy costs for domestic consumers as well as the investment costs for the network operator [3]. Moreover, residential storage units within a residential community can be exploited to decarbonize the grid [4]. In recent times, the success of energy trading and sharing schemes, such as EnergySage's solar energy community sharing project, deX's energy trading platform and Power Ledger's energy exchange scheme, have contributed to the motivation of researchers to investigate the application of similar sharing models for energy storage [2]. The authors of [3] proposed the shared ownership of energy storage resources between residential customers and network operators. To reduce the electricity bills incurred by homes within a smart grid, an energy storage capacity allocation algorithm was proposed in [5]. A virtualized energy storage sharing scheme was proposed in [6], which allows multiple users to virtually own and utilize a fraction of an energy storage unit. The authors of [7] proposed a distributed auction scheme for the allocation of multiple products (including charging and discharging power, energy and capacity) from energy storage resources within a residential community. The authors of [8] also proposed a smart contract-based 
energy storage sharing scheme for a joint energy storage market involving entities such as individual consumers, renewable energy plants and thermal power plants. In [9], an agent-based energy trading framework where energy storage systems are able to trade their services to support grid management is proposed. The model was found to be effective for reinforcing the demand-supply balance using a combination global and local energy storage systems. The authors of [10] investigated a grid comprising multiple renewable energy owners sharing an energy storage system (ESS). The results of their simulation showed that users profit considerably more from such sharing mechanisms compared to owning individual storage systems. Cloud energy storage is a concept that describes the logical sharing of an ESS by multiple users [11]. A technique that allows peer-to-peer (P2P) capacity sharing among users was studied in [11]. In [12], a cloud energy storage solution for utilizing distributed energy storage systems in microgrids is presented. The authors of [13] propose a model for the management of shared energy storage underpinned by proxy signatures in a blockchain setting.

Despite the benefits of energy storage sharing highlighted above, the centralized systems used to implement some of these schemes have drawbacks. Transaction clumsiness and data privacy are some issues associated with centralized databases [14]. Management inefficiencies and high transaction costs can also result from the use of centralized systems for energy transactions [14]. An emerging decentralized technology already being employed to facilitate transactions between peers on a network is blockchain. While centralized systems are susceptible to cyber attacks, blockchains can still reach a consensus on the system's state while under attack [15]. Blockchain technology is being used to implement transparent, efficient and secureP2P transactions within and beyond the energy sector. User privacy and security can remain intact even when transactions over a blockchain are public [16]. The technology promises a low cost and high computing power in energy trading schemes [17]. Many blockchain applications are made possible largely due to the incorporation of smart contracts. Smart contracts coupled with blockchain can enable credible P2P transactions [14].

Blockchains find applications in a number of fields within the energy industry. In [18], an integrated platform that implements energy management alongside a bilateral trade mechanism was proposed, which employs blockchain. Smart contract technology was utilized for energy management in a distributed energy environment in [19]. A blockchainbased, decentralized solution to the optimal power flow problem was proposed in [20].

Blockchain technology is also being widely adopted to facilitate energy trading [21]; in fact, there are existing real-world implementations of its application in this area. A popular application is the Brooklyn microgrid, where prosumers are able to sell surplus solar energy to their neighbors. A blockchain-based, Industrial-Internet-of-Things-assisted energy trading scheme was proposed in [22]. The authors of [23] also developed a blockchain-based P2P energy transactions framework for Industrial Internet of Things transaction scenarios.

While small-scale storage units can be easily purchased by homeowners who are motivated by the economic benefits they can potentially provide, large-scale units, which are required by large consumers such as shared facility controllers (SFCs), can be quite expensive and require a large physical space. SFCs are entities conferred with the responsibility to manage shared facilities within a community [24]. Examples of such shared facilities are parking lots, central water pumping systems and elevators. In [4], an auction-based mechanism was used to facilitate the sharing of residential storage units (RSUs) between the unit owners and SFCs.

This present paper is mainly motivated by the work presented in [4]. However, the auctioneer in their scheme is a central, third-party entity, which introduces the issues regarding a single point of failure, additional cost and the need to trust the entity to operate according to the pre-defined rules. In contrast to the work in [4], we propose the use of a decentralized scheme powered by blockchain technology. A blockchain-based smart contract is developed to facilitate an auction procedure for sharing storage capacities between RSUs and SFCs. The rules of the auction, which all the involved parties consent 
to, are programmed into the smart contract and can be audited by any of the parties. Moreover, funds are received in the smart contract from SFCs, and payments are made from the smart contract to RSUs in a cryptographically secure manner. Although the authors of [8] already proposed a smart contract-based energy storage sharing scheme, while we consider a market comprising SFCs and RSUs, they considered a joint energy storage market. Moreover, unlike our scheme, Substrate private blockchain was used for their smart contract development.

The remainder of this paper is organized as follows. The concepts of blockchain and a smart contract are explained in Section 2, followed by the description of a smart contractbased auctioning architecture in Section 3. Section 4 details the proposed auction-based energy storage scheme programmed into the smart contract and the flowchart/algorithm of the scheme. The scenarios investigated are presented in Section 5, and the results obtained are discussed in Section 6. Section 7 concludes the paper.

\section{Overview of Blockchain and Smart Contract}

Following the deployment and remarkable success of Bitcoin, blockchain technology began to draw much attention from the public. Bitcoin is a notable cryptocurrency that employs blockchain. It is a global network of peers who can securely send 'coins' to one another without third-party engagement. The network operates in a decentralized manner, utilizing cryptography and consensus mechanisms. Despite being an outstanding innovation, Bitcoin is only a finance-focused application of blockchain. Blockchain possesses huge potential beyond finance, especially with the incorporation of smart contracts. As a consequence of Bitcoin, there are numerous other blockchains that promise various improvements to the Bitcoin blockchain. The Ethereum blockchain, which includes the smart contract functionality, is a prevalent blockchain. A smart contract is a set of conditions or instructions that are digitally represented and executed on a blockchain. According to the Ethereum white paper [25], smart contracts are "systems which automatically move digital assets according to arbitrary pre-specified rules". Being underpinned by blockchain, smart contracts are executed in a decentralized manner and benefit from the cryptographic security mechanism of blockchains. With their ability to enforce coded agreements between multiple parties, smart contracts have the potential to eliminate intermediaries in wide range of applications. They are also able to receive and hold cryptocurrencies, and hence are capable of offering escrow services. The scope of applications of blockchains is significantly extended with the introduction of smart contracts. Ethereum contracts can be written in the Solidity or Vyper programming languages.

\section{Smart Contract-based Auctioning Architecture}

Inspired by the work presented in [26], Figure 1 shows a simplified smart contractbased architecture for storage sharing among RSUs and SFCs. These entities are each equipped with a smart meter, and communication is enabled between the meters and a smart contract. RSUs and SFCs are also able to interface with the smart contract via a desktop or mobile application. RSUs that are willing to share storage capacity send their offers (in terms of capacity and cost) to the smart contract, while SFCs in need of storage capacity send their bids along with funds to the smart contract. The contract performs the auction to determine the RSUs and SFCs who will be selling/buying storage capacities. Payment is then made to RSUs that fulfil their obligations. 


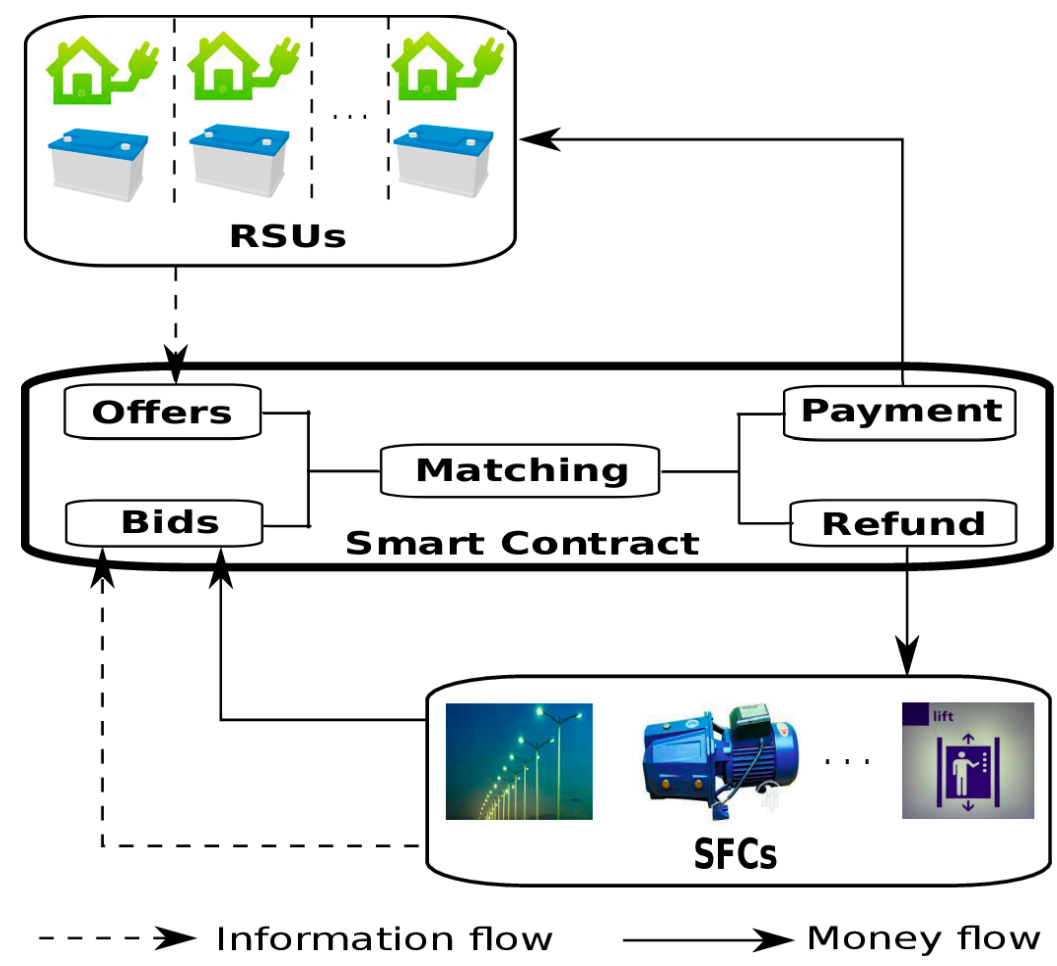

Figure 1. Smart contract-based auctioning for energy storage sharing.

\section{Proposed Smart-Contract-Based Storage Capacity Sharing Mechanism}

As shown in Algorithm 1, the proposed smart contract is made up of functions with varying access rights. Key functions are only accessible to the account address of the administrator, while other functions (except Function 1) are freely accessible. It should be noted that the calls made by the administrator to some of these functions can be automated in the practical implementation of the scheme. Function 1 is only executed at the point of contract deployment, and assigns the account address of the contract deployer to a variable. This variable is stored on-chain and used to grant or prohibit access to some functions. Function 2, for instance, can only be called by the deployer of the contract. It is called to set the dollar equivalent of one ether, which is the native currency of the Ethereum blockchain. Function 3 is called by SFCs intending to make bids for storage capacity, in order to view the ether equivalent of their bid. RSUs call Function 4 to submit their offers, stating RSU's storage capacity (RSC) in kWh and storage price (RSP) in USD/kWh. These data, along with their account addresses, are stored on-chain, and used for the verification carried out in the following function. In line with the double auction procedure, the data are exported for reordering and sent back to Function 5. The account address, storage capacity and offer price of each RSU are passed as input argument to Function 5, which checks that they match the original on-chain data, and then stores them in the new order. Function 6 receives SFCs' bid capacity (SBC), bid price (SBP) and funds the commensurate with the bid. The account addresses of SFCs, SBCs and SBPs are passed as input argument to Function 7, which checks that the data match the original on-chain data, and then stores them in the new order. To execute the matching of RSUs and SFCs based on the auction procedure programmed into the smart contract, a call to Function 8 is made. A flowchart that depicts the matching algorithm is shown in Figure 2. After the auction round, successful RSUs are paid by calling Function 9 (if they fulfil their storage capacity offering), while unsuccessful SFCs are refunded with Function 10. 


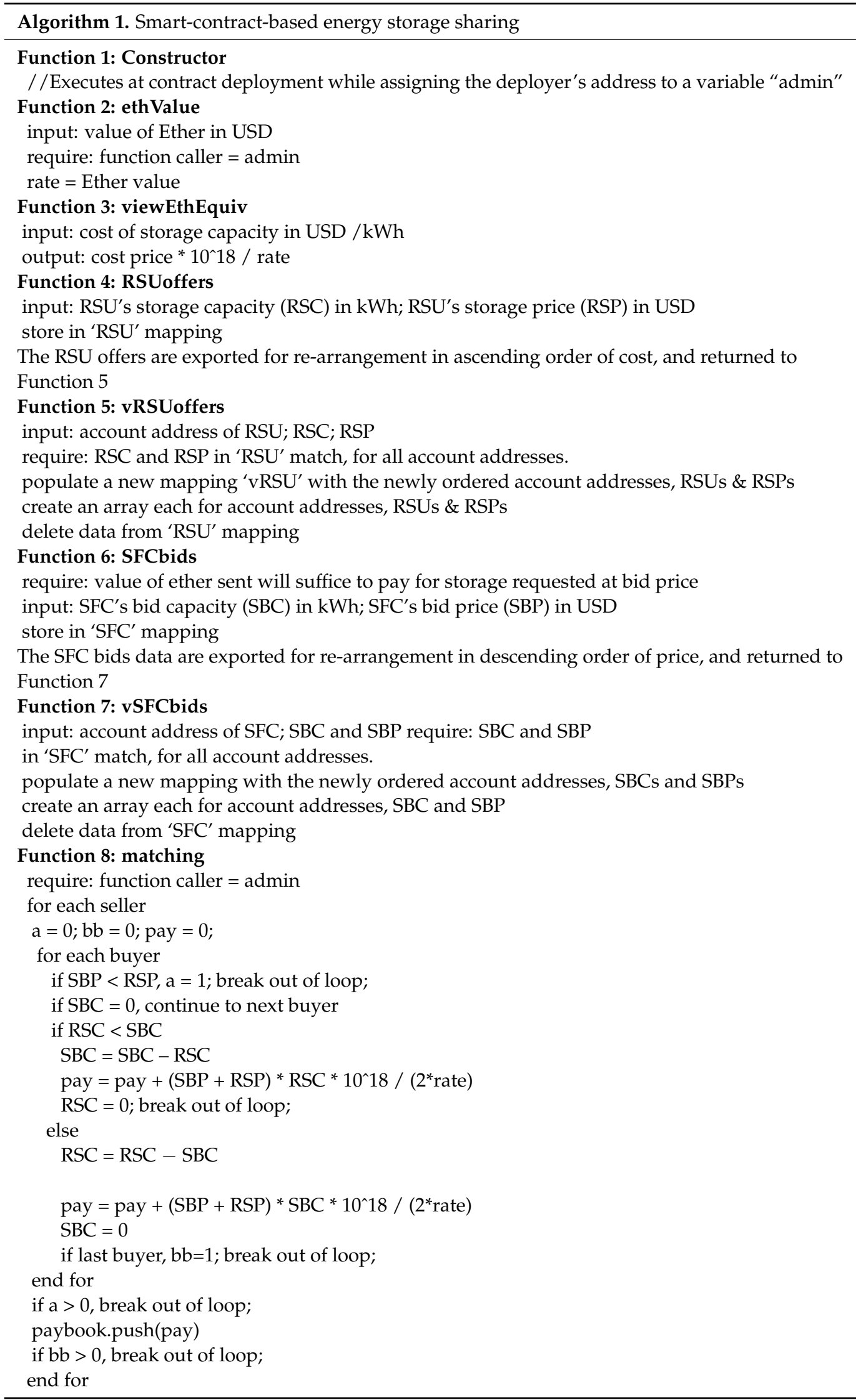



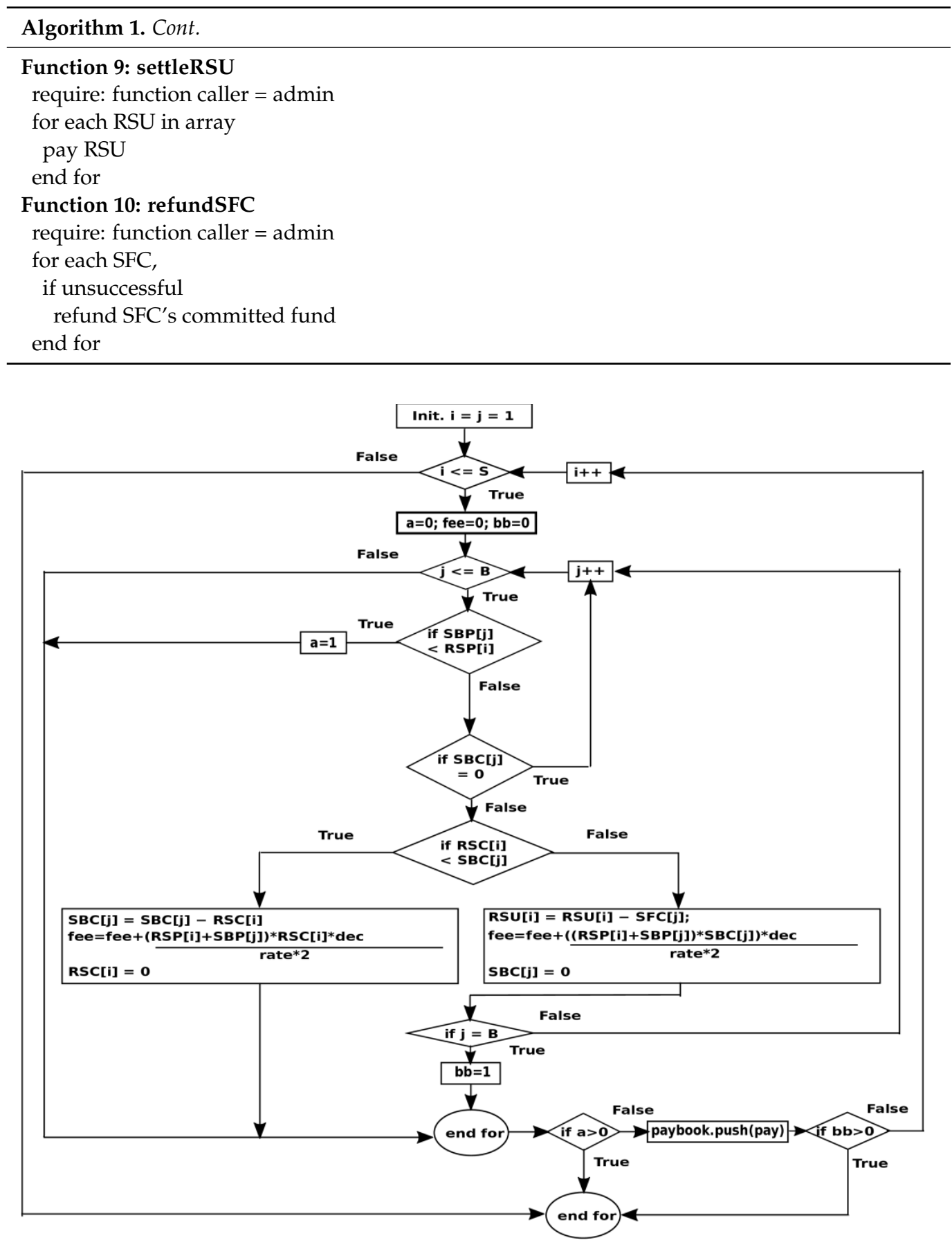

Figure 2. Flowchart of the matching function of the energy storage sharing scheme.

\section{Scenario Testing}

Inspired by the numerical case study in [27], we consider aggregated RSUs within a smart community that are willing to share storage capacity with SFCs in the community. Two scenarios are studied: Scenario 1-battery capacity supply exceeds demand and Scenario 2-capacity demand outweighs supply. Six groups of RSUs and five SFCs are assumed for Scenario 1, whereas five groups of RSUs and six SFCs are considered for Scenario 2. Their storage capacity offers/bids are randomly generated in the ranges from 100 to $500 \mathrm{kWh}$ at prices within the range from USD 20 to 70 . Tables 1 and 2 show offer/bid data for RSUs and SFCs in terms of capacity and price. Six RSU groups would be sufficient to cover a large community since each could comprise a sizeable number of households. Additionally, as the type of storage unit can vary widely, so should the RSU prices, hence 
the wide range used above. The foregoing data would suffice to test the auction logic implemented by the smart contract, as well as the accuracy of payments/refunds made to the concerned entities. It should be noted that all the parameter values assumed above are particular to the present work only. Depending on the season of the year, country, trading policy, number of willing RSUs and SFC requirements, these values may vary. The numerical test is performed using an Intel core i5 processor, 7.7GiB memory PC running Ubuntu 20.04.1 LTS operating system. The smart contract is written with Solidity, and deployed in the Remix-integrated development environment.

Table 1. Offer/bid data for RSUs and SFCs in terms of capacity and price (Scenario 1).

\begin{tabular}{cccccc}
\hline \multicolumn{2}{c}{ Aggregated Residential Storage Unit Offers } & \multicolumn{3}{c}{ Shared Facility Controller Bids } \\
\hline RSU Group & Offer (USD/kWh) & Capacity (kWh) & SFC & Bid (USD/kWh) & Capacity (kWh) \\
\hline A & 43 & 167 & a & 51 & 300 \\
B & 33 & 475 & b & 48 & 366 \\
C & 47 & 474 & c & 58 & 225 \\
D & 69 & 143 & d & 56 & 171 \\
E & 53 & 449 & e & & \\
F & 62 & 353 & & & \\
\hline
\end{tabular}

Table 2. Offer/bid data for RSUs and SFCs in terms of capacity and price (Scenario 2).

\begin{tabular}{cccccc}
\hline & Shared Facility Controller Bids & \multicolumn{2}{c}{ Aggregated Residential Storage Unit Offers } \\
\hline SFC & Bid (USD/kWh) & Capacity (kWh) & RSU Group & Offer (USD/kWh) & Capacity (kWh) \\
\hline A & 43 & 167 & a & 51 & 300 \\
B & 33 & 475 & b & 48 & 366 \\
C & 47 & 474 & c & 58 & 225 \\
D & 69 & 143 & d & 56 & 171 \\
E & 53 & 449 & & & \\
F & 62 & 353 & & & \\
\hline
\end{tabular}

\section{Results and Discussion}

Figure 3 shows the offers and bids submission by RSUs and SFCs, respectively. It also details the matching executed by the smart-contract-based on the double auction mechanism programmed into it. RSUs and SFCs both submit their offers and bids, respectively, to the contract; however, along with bids, SFCs send funds equivalent to their bids. In line with the auction procedure, offers are arranged in ascending order of price, while bids are arranged in the reverse order. The resulting ordered lists are: RSU - B, A, C, E, F, and D; SFC $-d, e, a, b$ and c. Following this, the matching of RSUs and SFCs is carried out. This begins from the tops of the ordered lists with RSU B serving SFC d with its entire capacity offer at a price midpoint between the offer and bid prices [28]. Matching continues until an SFC's bid price is less than an RSU's offer price. This occurred after RSU C was matched with SFC b.

As can be seen in Figure 3, the offered storage capacities are allocated in a continuous manner to the bidding SFCs, that is, a single RSU can serve multiple SFCs and multiple RSUs can serve a single SFC. For instance, RSU A serves both SFCs d and e, and SFC d is served by both RSUs B and A. This is a more economic and efficient approach than its discrete counterpart. Moreover, in terms of practical implementation, the approach is feasible since the RSUs will be operated as a pool rather than as unique entities. 


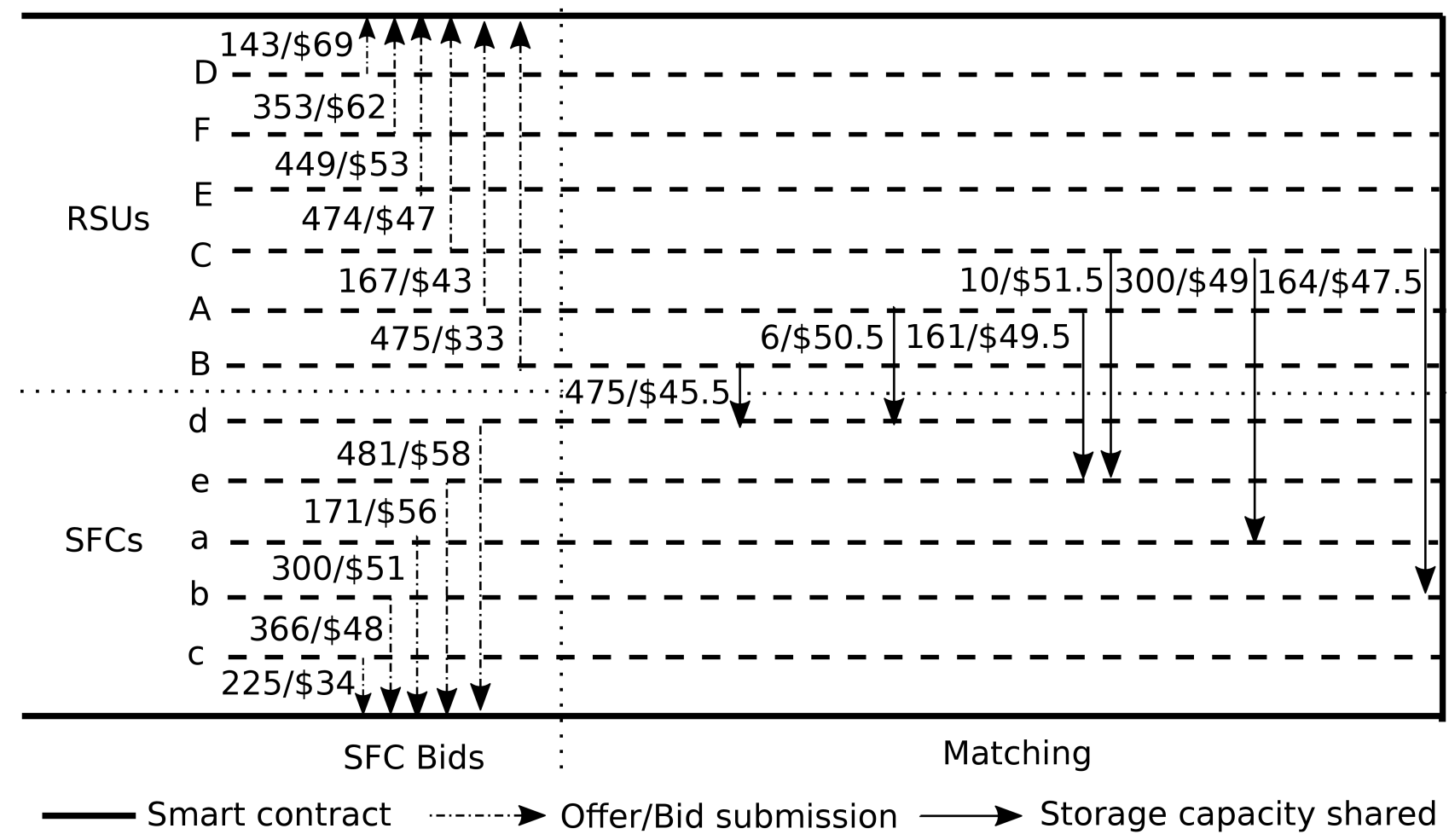

Figure 3. Smart contract execution of matching (Scenario 1).

Figure 4 shows the flow of funds between entities in the proposed scheme. While bidding, SFCs also send funds to the smart contract. Similar to an escrow account, the smart contract is able to hold funds until certain conditions are met. The funds sent by the bidders are commensurate with their bids. Payment is made from the smart contract to successful RSUs, while SFCs who are either partially or fully unsuccessful are refunded accordingly. From Figure 4, only RSUs B, A and C were paid by the contract, as these were the only successful RSUs. Based on their storage capacity allocations after the auction round, a full refund was made to SFC $c$, who received no allocation at all, while SFC $b$ received a partial refund, having received partial allocation. There is, however, an issue that is open to further investigation - the payment of RSUs and refunds to SFCs, which are blockchain transactions initiated from the smart contract and cost a certain amount of wei (a fraction of an ether). Additionally, wei is also spent while calling some of the other functions within the smart contract. This wei is used to incentivize the miners who secure the blockchain network. There needs to be a mechanism that fairly distributes this cost among all the entities partaking in the storage-sharing scheme.

Scenario 2 simulates a situation where battery capacity demand exceeds supply. Similar to the first scenario, RSUs/SFCs submit their offers/bids to the smart contract. Following the rearrangement of offers and bids in ascending and descending orders, respectively, the contract matches them based on pre-defined rules. From Figure 5, it can be observed that only RSUs c, b and a trade capacities with SFCs D, F and E. Although RSUs e and d have available capacities, their offer prices (USD 56 and USD 58) are higher than the available bid prices of E, C, A and B (USD 53, USD 47, USD 43 and USD 33). Figure 6 shows the payments made to/from the smart contract. The contract can again be seen to accurately match market entities, pay sellers and refund unsuccessful buyers. 


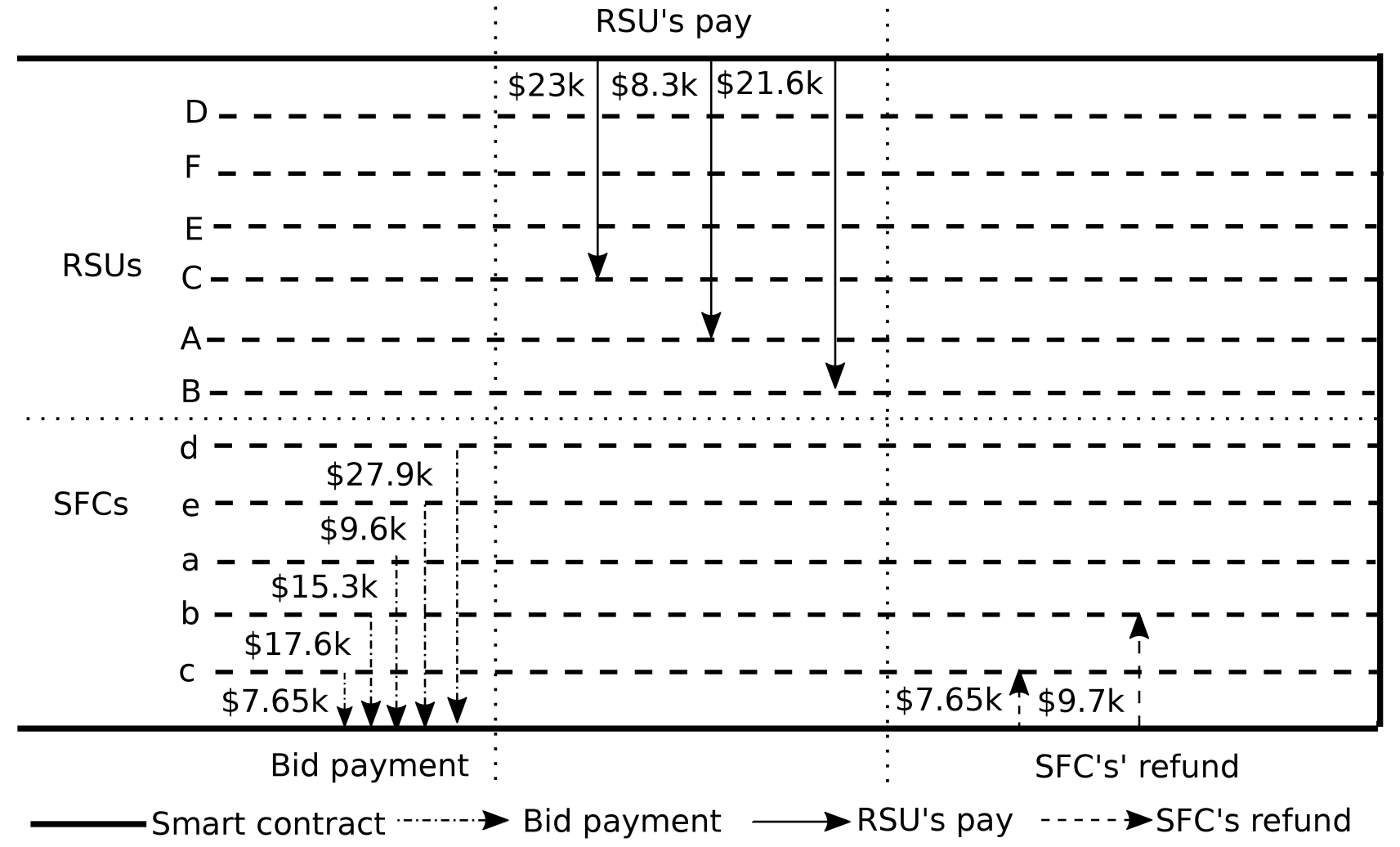

Figure 4. Smart contract execution of payments (Scenario 1).

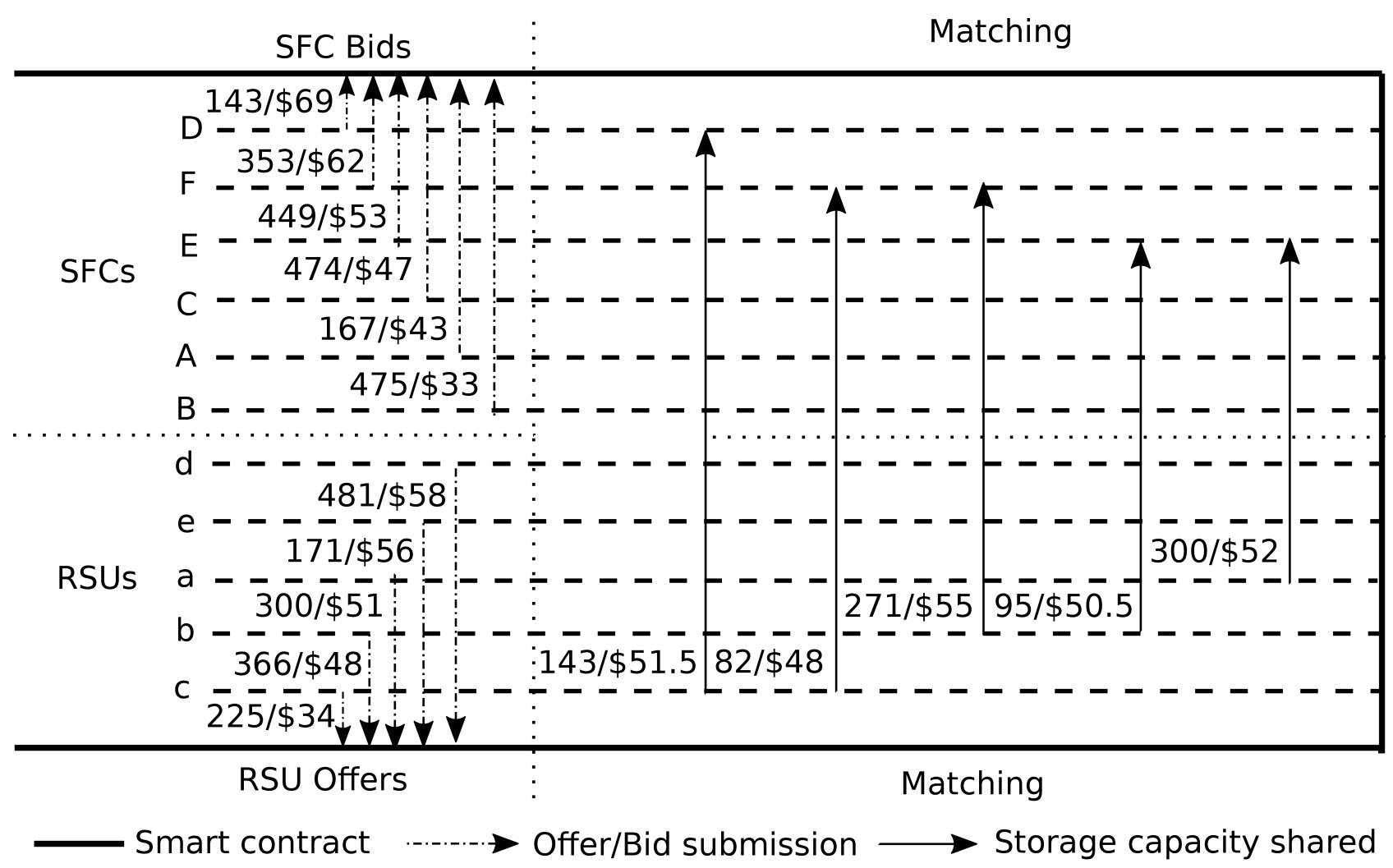

Figure 5. Smart contract execution of matching (Scenario 2) 


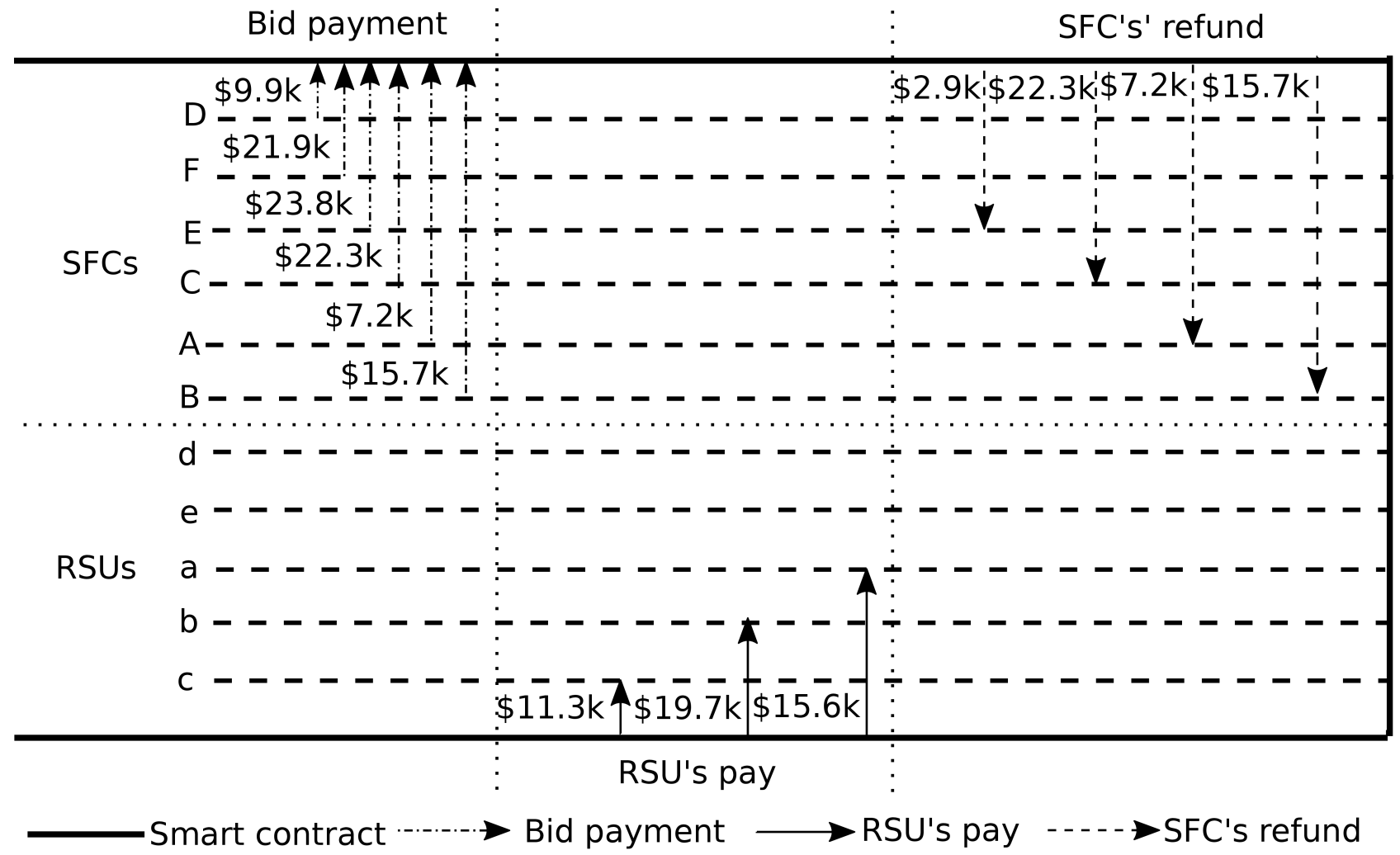

Figure 6. Smart contract execution of payments (Scenario 2).

Whereas the authors of [4] employed a centralized scheme for their auction-based energy storage mechanism, a blockchain-based decentralized scheme is proposed in this study. Centralized schemes can be prone to the single-point-of-failure issue and cyber attacks. Furthermore, third-party centralized authority needs to be trusted to act according to pre-defined rules. On the contrary, our proposed decentralized scheme is a smartcontract-based scheme meant to be implemented on the Ethereum blockchain. Being a public blockchain, smart contracts and transactions on Ethereum are cryptographically secured and fully transparent. In [8], a private blockchain (Substrate) was employed to facilitate energy storage sharing in a joint energy storage market. While private blockchains can be relatively more efficient and faster than public chains, they tend to be operated in a somewhat centralized manner.

\section{Conclusions}

Being underpinned by the cryptographic security mechanisms of the blockchain technology, smart contracts can be trusted to function as programmed without centralized control. Moreover, similar to an escrow account, it can also securely store funds until certain conditions are met. In this paper, an Ethereum-based smart contract was developed to perform a double-auction storage-sharing procedure. This procedure accepts offers from RSUs and bids from SFCs, matches them and then facilitates payments after obligations have been met. Scenario tests carried out on the smart contract show that it can effectively perform a double-auction procedure and make appropriate payments and refunds. With the successful implementation of such storage-sharing schemes, SFCs are able to leverage storage without having to make huge capital investments, while RSUs can exploit their units for financial gains. The increased investment in energy storage, which can improve grid management, is another potential consequence of successfully implementing storagesharing schemes.

The sharing of RSUs for the provision of ancillary services via an auction-based, smart-contract-enabled scheme can be explored in a further study. Additionally, a de- 
tailed comparison of the cost of engaging an auctioneer, with the cost of deploying and transacting with a smart contract, can constitute the motivation for another study. With respect to the auction approach adopted, a further study could examine the battery capacity surplus/deficit after an auction round by means of sensitivity analysis (e.g., Monte Carlo simulation).

Author Contributions: Conceptualization, U.D.; methodology, U.D.; software, U.D.; validation, U.D.; investigation, U.D.; writing—original draft preparation, U.D.; writing—review and editing, U.D. and N.I.N.; supervision, N.I.N.; project administration, N.I.N.; All authors have read and agreed to the published version of the manuscript."

Funding: This research received no external funding.

Data Availability Statement: Not applicable.

Conflicts of Interest: The authors declare no conflict of interest.

\section{References}

1. Chakraborty, P.; Baeyens, E.; Poolla, K.; Khargonekar, P.P.; Varaiya, P. Sharing Storage in a Smart Grid: A Coalitional Game Approach. IEEE Trans. Smart Grid 2019, 10, 4379-4390. [CrossRef]

2. Dai, R.; Esmaeilbeigi, R.; Charkhgard, H. The Utilization of Shared Energy Storage in Energy Systems: A Comprehensive Review. IEEE Trans. Smart Grid 2021, 12, 3163-3174. [CrossRef]

3. Wang, Z.; Gu, C.; Li, F.; Bale, P.; Sun, H. Active Demand Response Using Shared Energy Storage for Household Energy Management. IEEE Trans. Smart Grid 2013, 4, 1888-1897. [CrossRef]

4. Tushar, W.; Chai, B.; Yuen, C.; Huang, S.; Smith, D.B.; Poor, H.V.; Yang, Z. Energy storage sharing in smart grid: A modified auc-tion-based approach. IEEE Trans. Smart Grid 2016, 7, 1462-1475. [CrossRef]

5. Xie, K.; Zhong, W.; Li, W.; Zhu, Y. Distributed Capacity Allocation of Shared Energy Storage Using Online Convex Optimization. Energies 2019, 12, 1642. [CrossRef]

6. Zhao, D.; Wang, H.; Huang, J.; Lin, X. Virtual energy storage sharing and capacity allocation. IEEE Trans. Smart Grid 2019, 11, 1112-1123. [CrossRef]

7. Zhong, W.; Xie, K.; Liu, Y.; Yang, C.; Xie, S. Multi-Resource Allocation of Shared Energy Storage: A Distributed Combinatorial Auction Approach. IEEE Trans. Smart Grid 2020, 11, 4105-4115. [CrossRef]

8. Luo, B.; Shen, X.; Ping, J. Energy Storage Sharing Mechanism Based on Blockchain. In Proceedings of the 2020 IEEE 3rd Student Conference on Electrical Machines and Systems (SCEMS), Jinan, China, 4-6 December 2020; pp. 913-917.

9. Nunna, H.K.; Sesetti, A.; Rathore, A.K.; Doolla, S. Multiagent-based energy trading platform for energy storage systems in distribution systems with interconnected microgrids. IEEE Trans. Ind. Appl. 2020, 56, 3207-3217. [CrossRef]

10. Rahbar, K.; Moghadam, M.R.V.; Panda, S.K.; Reindl, T. Shared energy storage management for renewable energy integration in smart grid. In Proceedings of the 2016 IEEE Power \& Energy Society Innovative Smart Grid Technologies Conference (ISGT), Minneapolis, MN, USA, 6-9 September 2016; pp. 1-5.

11. Sim, J.; Kim, M.; Kim, D.; Kim, H. Cloud Energy Storage System Operation with Capacity P2P Transaction. Energies 2021, 14, 339 [CrossRef]

12. Saini, V.K.; Gupta, V.; Kumar, R.; Panigrahi, B.K.; Mahmud, M.A. Cloud Energy Storage Systems for Consumers and Prosumers in Residential Microgrids. In Proceedings of the 2020 IEEE International Conference on Power Electronics, Drives and Energy Systems (PEDES), Jaipur, India, 16-19 December 2020; pp. 1-6.

13. Wang, Y.; Qiu, W.; Dong, L.; Zhou, W.; Pei, Y.; Yang, L.; Nian, H.; Lin, Z. Proxy Signature-Based Management Model of Sharing Energy Storage in Blockchain Environment. Appl. Sci. 2020, 10, 7502. [CrossRef]

14. Han, D.; Zhang, C.; Ping, J.; Yan, Z. Smart contract architecture for decentralized energy trading and management based on blockchains. Energy 2020, 199, 117417. [CrossRef]

15. Munsing, E.; Mather, J.; Moura, S. Blockchains for decentralized optimization of energy resources in microgrid networks. In Proceedings of the 2017 IEEE Conference on Control Technology and Applications (CCTA), Maui, HI, USA, 27-30 August 2017; pp. 2164-2171.

16. Abdella, J.; Shuaib, K. Peer to Peer Distributed Energy Trading in Smart Grids: A Survey. Energies 2018, 11, 1560. [CrossRef]

17. Wang, N.; Zhou, X.; Lu, X.; Guan, Z.; Wu, L.; Du, X.; Guizani, M. When Energy Trading Meets Blockchain in Electrical Power System: The State of the Art. Appl. Sci. 2019, 9, 1561. [CrossRef]

18. Van Leeuwen, G.; AlSkaif, T.; Gibescu, M.; van Sark, W. An integrated blockchain-based energy management platform with bilateral trading for microgrid communities. Appl. Energy 2020, 263, 114613. [CrossRef]

19. Li, Y.; Yang, W.; He, P.; Chen, C.; Wang, X. Design and management of a distributed hybrid energy system through smart contract and blockchain. Appl. Energy 2019, 248, 390-405. [CrossRef]

20. Foti, M.; Mavromatis, C.; Vavalis, M. Decentralized blockchain-based consensus for Optimal Power Flow solutions. Appl. Energy 2021, 283, 116100. [CrossRef] 
21. Guo, J.; Ding, X.; Wu, W. Combined Cooling, Heating, and Power System in Blockchain-Enabled Energy Management. arXiv 2020, arXiv:2003.13416.

22. Guan, Z.; Lu, X.; Wang, N.; Wu, J.; Du, X.; Guizani, M. Towards secure and efficient energy trading in IIoT-enabled energy internet: A blockchain approach. Future Gener. Comput. Syst. 2020, 110, 686-695. [CrossRef]

23. $\mathrm{Hu}, \mathrm{W}$.; Li, H. A blockchain-based secure transaction model for distributed energy in Industrial Internet of Things. Alex. Eng. J. 2021, 60, 491-500. [CrossRef]

24. Tushar, W.; Chai, B.; Yuen, C.; Smith, D.B.; Wood, K.L.; Yang, Z.; Poor, H.V. Three-Party Energy Management with Distributed Energy Resources in Smart Grid. IEEE Trans. Ind. Electron. 2015, 62, 2487-2498. [CrossRef]

25. Buterin, V. A next-generation smart contract and decentralized application platform. White Pap. $2014,3,37$.

26. Hua, W.; Jiang, J.; Sun, H.; Wu, J. A blockchain based peer-to-peer trading framework integrating energy and carbon markets. Appl. Energy 2020, 279, 115539. [CrossRef]

27. Fang, D.; Wu, J.; Tang, D. A double auction model for competitive generators and large consumers considering power transmission cost. Int. J. Electr. Power Energy Syst. 2012, 43, 880-888. [CrossRef]

28. Nicolaisen, J.; Petrov, V.; Tesfatsion, L. Market power and efficiency in a computational electricity market with discriminatory double-auction pricing. IEEE Trans. Evol. Comput. 2001, 5, 504-523. [CrossRef] 